\title{
36
}

\section{Experiences With the OSF Distributed Computing Environment}

\author{
John Dilley, Hewlett-Packard Laboratories
}

This paper describes the Open Software Foundation's Distributed Computing Environment (OSF DCE) in the context of the ISO Reference Model of Open Distributed Processing (RMODP). It presents a critical assessment of the DCE technology, and suggests future work for both DCE and ODP. We suggest how DCE can support Open Distributed Processing, summarize the experiences we had building a DCE-based prototype, and discuss the lessons we learned from this activity. As a result of the prototype effort we built a set of DCE services to improve the usability and utility of DCE, including an object-oriented programming system, an event notification service, and a name space browser/editor for the DCE directory service. Current research efforts are underway to study fault tolerance and to provide generic data replication.

Keyword Codes: C.2.4; C.2.2; D.1.5

Keywords: Distributed Computing; Network Protocols; Object-Oriented Programming

\section{BACKGROUND}

In 1991 Hewlett-Packard's Networked Systems Architecture group began to develop prototype software to learn more about the Open Software Foundation's Distributed Computing Environment (OSF DCE). The prototypes were done in the context of HP's Cooperative Computing Environment (CCE [1]) Architecture Framework. Many of the goals of the CCE framework were similar to those of the ISO ODP effort, described in [2][3][4][5], and summarized in a very readable way in [6]. The structure of the CCE framework was based upon the ISO ODP viewpoints, applied to a constrained set of objectives that focused on HP's business interests. Both efforts defined a set of crucial services and mechanisms required in a distributed environment and a vocabulary for describing distributed components, applications, and systems.

At that time the ODP viewpoints did not consider legacy environments, which made it diffcult to support HP's business. The ODP viewpoints were also not focused on any business areas. In trying to apply the descriptions of the Enterprise and Information viewpoints to HP's CCE we concluded that the Enterprise viewpoint described a business model and would be hard to generalize and still be a useful model for application design. For our CCE prototype, the Enterprise viewpoint became a set of requirements to support business processes and assumptions.

The Information model seemed to overlap with the Enterprise and Computational viewpoints, therefore we developed our own model for information. The model centered on a particular information system and its application: information was described in four aspects, Input, Output, Presentation and Storage. Within each of these aspects there were attributes such as Security and Consistency that would need to be supported at the Engineering level.

The Computational viewpoint was well defined but based around a linguistic framework (ANSA DPL). Although most agreed that this would be the ideal way to model the Computa- 
tional viewpoint, it did not reflect reality-since programmers did not want to learn another language. HP had an installed base that could not be moved to a new linguistic framework and therefore needed a more evolutionary approach to the computational model.

The CCE computational viewpoint described the interaction model and development model for distributed computing systems and identified development and runtime services that would be required to support the viewpoint and integrate the installed base of applications and system level services that had been written in a multitude of languages and would not be re-written.

\section{OSF DCE OVERVIEW}

The Open Software Foundation's Distributed Computing Environment (OSF DCE) is a software infrastructure for development of distributed computing applications. DCE facilities are accessed through programmatic and RPC interfaces, both defined in the DCE Application Environment Specification (AES [7]); some of the services are also accessible through user-level commands.

The OSF took a different approach than the ODP group at defining an open distributed computing environment. The DCE provides a set of distributed computing technologies, and uses those technologies to define its distributed environment. On the one hand this does not provide the high-level aspects provided by ODP, such as a common vocabulary for specifying viewpoints for distributed computing. On the other hand, DCE provides specific software technology, with a reference (or sample) implementation, assisting application portability and assuring interoperability. Since ODP does not currently specify a technology viewpoint (i.e., it does not provide strict specifications for APIs or wire protocols), it is likely that early "ODP-conformant" applications will not interoperate.

The components of DCE are summarized in relation to ODP in the following sections.

\subsection{Communications}

The Remote Procedure Call (RPC) component allows client processes to communicate with servers across a local or wide-area network. DCE RPC uses the procedure call/return paradigm for remote communication-a remote procedure call looks to the developer like a local procedure call, however there are some significant differences in call latency and fault semantics which developers need to be aware of when writing RPC applications.

RPC development starts with the definition of data types and remotely-callable procedures associated with an application. These types and operations are specified using the DCE Interface Definition Language (IDL). A compiler provided with DCE converts IDL specifications into RPC communication stubs. DCE RPC stubs provide for data marshaling, which prevents the application developer from having to explicitly handle data conversion between different systems. In RPC, parameters in remote operations, which can be complex data types (including pointers), are automatically traversed and converted into and out of a network transmissible form. RPC provides access transparency, as defined by ODP in [3][6].

\subsection{Directory Services}

Each remotely accessible object in a distributed system has a distinct network location which can vary over time. To access an object (or service) requires first determining its network location using some type of location or directory service. The benefit a directory service provides is 
that service locations do not need to be known at compile time (and statically bound), or even at program load time - but rather can be dynamically located, and even relocated at run time.

The DCE includes facilities called the Cell Directory Service (CDS) ${ }^{1}$ and Global Directory Service (GDS). The CDS is a cell-local directory service, whose main purpose is to provide a name-to-address lookup to allow clients to locate servers in the cell. DCE RPC includes a Name Service Interface (NSI), which clients use to access server location information in the CDS name space. NSI allows DCE clients to locate servers at run-time in a location-independent manner. In addition, the directory supports access to arbitrary information via the X.500 Directory Service (XDS [8]) interface. GDS allows multiple DCE cells to be connected to allow name space searches to traverse cell boundaries.

The directory service and the RPC runtime library together provide location transparency. An additional aspect of object location that ODP specifies is trading [9]. The DCE CDS does not provide a full constraint-based trading capability, however the NSI search facility effectively provides trading based upon the interface (type), object identity, and desired network transport protocol the client can use. We have found in most cases this is sufficient, though a full Trader in DCE would provide additional value.

\subsection{Time}

The DCE Distributed Time Service (DTS) maintains a single, global virtual clock, which is then used to keep machine clocks synchronized across the network. A consistent global time is required by the DCE Security Service authentication mechanism and the Distributed File System. DTS assures that each DCE cell will maintain a consistent, monotonically increasing time. Global DTS servers must be connected with an external time provider that provides the actual time signal. The DTS protocol then keeps host clocks consistent with Coordinated Universal Time (UTC). RM-ODP does not define an equivalent service [10].

\subsection{POSIX Threads}

The DCE Pthreads package defines a multi-tasking capability for distributed applications. DCE uses Pthreads to allow a single process to have many logical threads of control; these threads can initiate or serve RPCs concurrently. The Pthreads package provides synchronization primitives, including a mutual exclusion locking facility (mutex) which allows multiple threads to wait for a signal of a particular condition becoming true (condition variables). RM-ODP does not currently define a threading or concurrency model.

\subsection{Security}

Having an integrated security service is a key benefit of the DCE environment. Providing secure communications in systems that cannot trust all components of the network is quite difficult to achieve. The DCE Security Service, based upon MIT's Kerberos implementation, is fully integrated with the RPC mechanism. DCE Security provides mutual authentication of requests between client and server, so the client and server can be assured that each other's identity is valid. Based upon the trusted identity of a client, DCE applications can provide authorization of client requests to control access to resources using an Access Control List (ACL). The Security Service can utilize data encryption for RPC calls to prevent listeners on the network

1. A cell is the DCE unit of administrative division. 
from being able to intercept or tamper with RPC requests and responses. The DCE security system performs some of its security checks in the RPC run-time; certain authentication and authorization checks occur before user code is even invoked. Only if the caller is using a proper level of security does the call proceed into user code.

The DCE Security Service provides several of the security functions called for in ODP.

- Access Control Function is provided through DCE's Access Control List (ACL) facility.

- Security Audit Function is provided in the 1.1 release of DCE.

- Authentication Function is provided using the security service as the trusted third party, with principals stored in the DCE registry.

- Integrity Function is provided via authenticated RPC using encryption and checksums of data in RPC communications.

- Confidentiality Function is provided through the user data privacy option that encrypts data in RPC calls.

- Non-repudiation Function is not currently provided by DCE; there is no way to authoritatively convince a third party that a particular object was involved in an RPC. (The client and server are of course convinced.)

- Key Management Function is provided by DCE, in a way that relies upon the host file system security.

\subsection{Distributed File System}

DCE includes a Distributed File System (DFS), which provides a shared global file system visible to hosts in all connected cells. The DFS provides all users with the same view of the global file system; other distributed file systems present a different view of files depending upon whether one is on a file server or a client system. DFS uses file caching so that once a remote file is accessed, a copy is cached locally to improve performance. RM-ODP does not specify a distributed file system.

\section{THE DCE PROTOTYPE}

In 1991-1992, our group built a multimedia mail application to experiment with the fledgling Distributed Computing Environment [11][12]. Our intention was not to create a productionquality mail system, but rather to learn more about DCE. The goals of the DCE prototype effort were as follows.

- Validate the Cooperative Computing Environment framework through actual experience.

- Demonstrate the advantages of DCE-based client/server distributed computing over more traditional forms of distributed computing.

- Determine the aspects of distributed computing for which DCE was well suited. No distributed computing platform will provide an exact match for the requirements of every application; we wanted to figure out the tasks for which DCE was well suited, and those for which DCE did not do so well.

- Characterize DCE performance. One key aspect of a distributed environment is its implications on system and application performance. A key part of the prototype effort involved understanding the performance of DCE RPC the DCE directory service [13]. 
- Learn about management of distributed systems and applications. Operation and management of distributed applications is inherently more complex than for non-distributed applications.

- Identify areas where DCE should be enhanced. The first release of any technology is bound to be limited-we wanted to determine those areas where additional DCE tools and services were required to support distributed application development.

- Propose enhancements to improve the quality of DCE. Since HP is a DCE vendor, we wanted to make sure our efforts could be used to improve the product offering from HP as well as OSF's DCE in general.

\subsection{The MultiMedia Mail System}

The application we chose to exercise DCE was a multimedia mail system that could compose and send messages containing text, graphics, and voice annotations. We felt this system would

\begin{tabular}{|c|c|c|c|c|}
\hline Applications & & $\begin{array}{l}\text { Mail } \\
\text { ervice }\end{array}$ & $\begin{array}{c}\text { Storage } \\
\text { Mgmt }\end{array}$ & $\cdots$ \\
\hline $\begin{array}{l}\text { Distributed } \\
\text { Object Svcs }\end{array}$ & \multicolumn{2}{|c|}{$\begin{array}{c}\text { Information } \\
\text { Access }\end{array}$} & $\begin{array}{c}\text { GUI } \\
\text { (Motif) }\end{array}$ & $\begin{array}{c}\text { GDMO } \\
\text { Compiler }\end{array}$ \\
\hline Conversion & FAX & Voice & Storage & GSMS \\
\hline Events & & & & \\
\hline
\end{tabular}

FIGURE 1. MultiMedia System Components require enough of the facilities of the infrastructure to provide valuable insight into DCE, distributed application design and development, and distributed application management.

The distributed system we built consisted of several major components. Each of these components used DCE to provide distribution of service to clients across the local area network. The components we built were as follows:

- The mail service implemented a user mailbox and used store-and-forward networking to transmit mail messages between users. The mailbox and mail submission protocols were defined in IDL and implemented using RPC.

There were two mail service user interfaces, one based upon Andrew Mail and the other a custom DCE-based application. Through this we learned how to wrap existing applications that were not written with DCE in mind.

- A Fax service provided a transport for mail messages and a way to send graphics or text images to users who did not have an email box. Fax images could be separately stored and used as components in mail messages.

- A voice service provided recording and playback of voice messages. Voice messages could be used as annotations in mail messages.

- A distributed information storage system provided storage and retrieval of bulk data from servers on the network, including text, graphics, and voice and fax annotations. Multimedia messages were often quite large, so custom file servers with large disk arrays were helpful for bulk information storage.

- A conversion service converted data among the formats required by the various interfaces. For example, the conversion server could convert from the Fax format to the format used in the mail GUI. This type of service is often embedded in, for example, a mail service; we 
wanted to make the conversion a generic service for use by mail, fax, or other system components, to allow openness and the ability to dynamically add new system services.

- The Event Service provided asynchronous notification of faults and status information for the services in the environment.

- A generic service management interface provided access to the state of the servers and services in the network, and allowed system managers to start, stop, and restart services. The Event Service was used to convey state change notifications from application components to the management service. An RPC to a per-node management application was used to start server instances.

- A configuration service allowed services to store configuration data for use at start-up and during execution. The configuration state was globally available; this facility provided a standard configuration interface, instead of requiring each service to have a custom interface to its configuration data.

\subsection{DCE Lessons Learned}

Some of the initial challenges of using DCE were a steep learning curve, difficulty of application development, and lack of debugging tools for developers. Furthermore, initial installation and configuration of the DCE environment was time consuming, and determining the health of DCE at that time was non-trivial. Finally, early DCE was not integrated with the existing operating system and distributed computing software we were using.

All of the interfaces in our system were defined using DCE IDL and accessed via DCE RPC. The RPC mechanism provides a powerful client/server communication model; RPC keeps the programmer from having to deal with location or distribution issues in the system. By defining formal interfaces, we found that applications were easier to enhance and evolve. Provided that the interface does not change, we were able to evolve applications from providing quicklydeveloped but incomplete capabilities, to providing more sophisticated implementations as time permits. On the down side, the RPC interface was not easy to learn and use.

The Cell Directory Service provides the host independence part of the location transparency available to RPC applications. The CDS allows easy reallocation of services to systems in the network: the service simply registers its location upon start-up after which new clients will be able to locate it. ${ }^{2}$ CDS does not provide the ability to trade for services based on an arbitrary set of constraints. Having a trader available in DCE would be beneficial. It also did not at that time provide tools to assist with name space maintenance. There was a graphical browser and a command-line interface to the directory, but the former only displayed directory contents; the latter allowed modification, but not in the context of the browser, so it was more difficult to use.

One of the key challenges of writing DCE-based applications turned out to be dealing with their multi-threaded nature. The difficulty was not so much in writing thread-safe code as in using existing system software that was not written to be thread-safe. Until such a time when system and third party software is thread-safe, DCE developers must use caution when using non-DCE libraries, and may have to protect unsafe calls using mutual exclusion (mutex) locks.

2. This is not service migration; migration typically involves relocation without the client faulting or having to restart. 


\section{DCE ENHANCEMENTS}

Based upon the areas of improvement we identified building prototypes, we proposed a set of contributions we could make to enhance the DCE offering. HP has developed many of these facilities and delivered them as part of subsequent DCE releases. As and when the ODP offering includes a technology platform, these types of enhanced services will likely be required.

\subsection{Distributed Debugging Environment}

DDE provides a graphical-based user interface for DCE application debugging. It is a threadaware debugger, meaning you can manipulate the state of the threads in your application. DDE lets you view the threads in your process, enable or disable threads for execution, cancel threads, as well as do a full set of regular debug activities. While DDE is not a truly distributed debugger (in the sense that you cannot single step across process boundaries and debug the server executing a client request), it goes a long ways towards making DCE applications easier to debug.

\subsection{Trace/Log Facility}

The Trace/Log Facility allows developers to instrument their code with trace statements at "interesting" points. When the trace statement is executed, it emits a line of output according to a predefined format, which includes the time of the trace (to millisecond precision), the thread which executed the statement, a trace identifier (each trace object can have a separate identifier in its output), and a user-defined message. Global time (UTC) is used in the trace output to allow traces from multiple processes in different time zones to be sorted into a single trace. This is necessary to see the order of certain operations. Each trace statement can identify a selectorlevel, which allows run-time control over which messages are emitted.

\subsection{Instrumented IDL Compiler}

The DCE IDL compiler generates client and server communication stubs that provide distribution transparency (data marshaling, sending network packets). Sometimes when debugging distributed applications it is nice to determine where in the RPC process the program was when it aborted (distributed programs sometimes terminate none too gracefully). The Instrumented IDL Compiler is a version of the DCE IDL compiler that adds tracing statements (using the facility described above) in key points in the stubs. When the program runs, the IDL compiler's trace selectors can be turned up such that they will emit trace messages when they enter and exit the communication stubs, when they begin and finish marshaling data, and when they begin and finish sending the request. Elapsed times are included in the trace output to provide an indication of how long each step in the process took.

\subsection{Sample Applications}

The sample applications are a set of programs that demonstrate the use of DCE by application developers. They attempt to show the various facilities of DCE, from a very simple "getstarted" application, to applications focused on each of the DCE facilities, to a more representative application that uses RPC, naming, threads, and security together. These applications are extensively commented with a discussion of each new DCE API routine being used, the parameters and their types, their return value, and a description of the expected operation. The sample applications can be read as tutorial information, or leveraged to create new applications. 


\subsection{Object-Oriented DCE Framework}

Two limitations of the early DCE were difficulty of use for developers, and lack of support for $\mathrm{C}++$. We felt that by encapsulating the facilities of DCE in a $\mathrm{C}++$ class library, we could provide greater ease of use than regular DCE. While this could have been done by adding a $\mathrm{C}$ library with wrappers for the cumbersome data structures and API routines, this would not have provided access for $\mathrm{C}++$ developers, and could not have provided the same value. One reason is that DCE, as originally designed, included an object-oriented model for accessing distributed objects. Also, object-oriented analysis and design techniques are well suited to the requirements of distributed computing and distributed application development.

HP OODCE provides a $\mathrm{C}^{++}$framework for DCE application developers [14]. OODCE consists of an enhanced IDL compiler, which converts interface definitions into $\mathrm{C}++$ classes, and a class library that encapsulates much of the complexity of the DCE programming environment. The classes generated by the enhanced IDL compiler inherit from framework classes provided in the OODCE library. These framework classes define the structure of object-oriented DCE applications, and provide a significant amount of code to interact with the DCE run-time environment, for example to register location information and to interact with the security subsystem. In addition to the framework classes, a set of $\mathrm{C}++$ utility classes are provided to make it more convenient to deal with regular DCE data types.

An earlier ICODP paper reported on the use of DCE and $\mathrm{C}^{++}$as a programming environment for ODP [15]. The focus of that work was on a direct mapping from ODIN to DCE and C++. This work by contrast uses standard DCE IDL for interface definition, and is focused on making the existing DCE easy to use.

\subsection{On-Line Help Facility}

The help files for the sample applications are delivered as a set of README files, but in addition these files have been integrated with the Visual User Environment (VUE) hypertext help facility. This allows users to search for topics by keyword, and locate individual lessons in the various sample applications.

\subsection{Enhanced CDS Browser/Editor}

Being able to graphically view and manipulate the contents of the directory service is an essential capability in any distributed environment. The CDS browser provides an X11-based interface to the CDS name space, displaying it as a tree of objects. Through the browser the user can see the various RPC server entries, groups, and profiles in the directory name space. The DCE includes a basic CDS browser with viewing capabilities. The enhanced browser/editor we provided added the ability to interact with CDS to add, remove, or modify the entries in the name space, and to view and change any ACLs associated with an object.

\subsection{System Administration}

A set of tools have been deployed to allow more powerful administration of the distributed environment, including central point software installation, cell configuration management built into the standard System Administration Manager (SAM) tool, a cell validation test suite, and a snapshot facility to help troubleshoot cell problems. The validation suite and snapshot facility help administrators determine if the cell is operational, and to assess the state of DCE servers. 
The DCE configurator provides a single point of configuration for a DCE cell; it can discover the current cell configuration as a starting point for reconfiguration. The configurator also provides a cell planning facility, allowing administrators to plan and pre-test a cell configuration before committing the changes.

\subsection{Legacy System Integration}

The first action a user takes on a system is to log in - to specify and validate their identity by giving a user name (login name or principal in DCE terms) and a secret password. The original release of DCE required users to validate themselves to the system and to DCE separately; but the current release of DCE provides for an integrated login, allowing the user to establish their identity only once, and have DCE credentials automatically refreshed whenever the user gives their password to the system (for example to unlock their display).

The DCE Distributed File System provides an advanced distributed file system; however, the ONC Network File System (NFS) is currently the most widely deployed distributed file system. In order to allow use of both file systems, HP has supplied an NFS-DFS gateway, which allows NFS clients to access a DFS file system. This gateway allows gradual migration to DCE: servers upgraded to DCE and DFS can run the NFS-DFS gateway to allow existing clients the same access to their data as before. New clients can use DFS directly to gain the addition benefits DCE/DFS provides.

\subsection{Event Service}

DCE does not have a standard asynchronous notification (event) service at the time of this writing. As part of the prototype we developed and used one to support distributed systems management. The model we used was subscribe/publish, where each agent interested in receiving event notifications sent a subscription request to an event service indicating the event type, severity, and application generating the event. The event service receives subscription requests and maintains a database of interested subscribers; it also receives asynchronous events and redistributes them to event clients based upon the subscription database.

Using this service, event producers do not have to be aware of the entities interested in receiving events, nor with the details of distribution of events to them (and having to deal with the fact that some of them may be unreachable), nor do they have to be aware of whether anyone is in fact interested in an event. Instead, producers send all events to the Event Service and forget about them. The Event Service is then responsible for sending the event to all interested event consumers. Furthermore, event consumers do not need to be aware of specifically where events are generated. Instead, they receive any event that is of the type and severity they requested a subscription to.

\subsection{Systems Management}

While polling the state of the system was an important part of our prototype, we noted that a more efficient approach was to manage by exception. In other words, instead of relying upon an occasional poll to tell us that a component had gone down or come back up, we used the Event Service for this purpose. This way there was minimal traffic on the network in the steady state, but when some potentially interesting event occurred, a notification was sent; management agents could register their interest in that event type and then receive the notifications when they occurred. 
While this is far from a full system management solution, it did enable us to determine at a crude level whether the system was functional, and if not, where the fault lay.

\subsection{Data Replication Architecture}

In a distributed environment, data that needs to be highly available is typically replicated in several locations, to protect against a single failure causing data or services to be unavailable. Our goal in the data replication area is to make it possible to develop application logic independent of the availability strategy. We believe that the availability requirements not only depend on the nature of the application but also on the operating environment in which it is deployed. For example, the desired failure recovery strategy of the CDS depends on what kind of applications are depending on its availability. OLTP developers have told us that absolute correctness and consistency between CDS database replicas is a requirement for their applications. In other words, all replicas must be synchronized so that no name space operations are lost if a master crashes before propagating updates to a read-only replica. By contrast, certain other mission critical applications would rather have automatic fail-over of a read-only slave into a writable master even if some entries are temporarily lost so that new services could continue to export their bindings and continue running while CDS recovers.

In this CDS example, it would be nice to be able to write the CDS namespace database logic independently from the mechanism which will handle replication, fault detection, and recovery. For that reason, we have drafted a data replication architecture to describe the components of a replication system in a general way. We designed a general-purpose interface to which applications can be coded. The value is that replication systems with different configurations and policies may be implemented behind the standard interface. That frees the application developer from having to care about replication and failure recovery. Choosing the appropriate replication mechanism can be done at link time. This approach also provides a consistent way for an application to access data replication.

\section{FUTURE DIRECTIONS}

There is still much work to be done in the area of enhancing DCE in the areas of system and application management, fault tolerance and data replication, and asynchronous notification. In addition, the DCE effort would benefit from the vocabulary defined by RM-ODP for definition of Enterprise, Information, and Computational viewpoints (DCE already provides equivalents for the Engineering and Technology viewpoints).

In addition, the ODP effort may be able to benefit from industry experiences such as ours with distributed computing and DCE. Some specific issues and areas which merit further study are listed below.

- Should DCE make use of the Enterprise, Information, and Computational viewpoints from RM-ODP? Can DCE be described in terms of Engineering and Technology viewpoints?

- Can ODP make use of DCE technologies to provide interoperation between ODP-compliant applications? Could ODP use the DCE Engineering and Technology viewpoints, if defined? Without a technology mapping, interoperation is difficult to achieve. This has been illustrated by the CORBA effort, which initially specified only an architecture, but is now working to address interoperability between implementations [16]. 
- Distributed systems and application management is an area of continued investigation. End users want to be able to determine how to get their task done-they should not be expected to be network management and protocol experts.

\section{ACKNOWLEDGMENTS}

We would like to thank Deborah Caswell for providing the information on fault tolerance, recovery, and data replication; and Jeff Morgan for providing the historical view of the CCE. Thanks also to the following for their review comments on this work: Lee Boswell, Bob Fraley, Richard Friedrich, Mickey Gittler, Marta Kosarchyn, Joe Martinka, Bob Price, Walt Tuvell. Thanks also to the pioneering members of the original "Exploitation of DCE" prototype team, and especially to the team that made HP's DCE into a viable distributed computing platform.

\section{REFERENCES}

[1] NewWave Computing Framework, Vol 1-3, Version 1.1. Hewlett-Packard, Information Architecture Group, October 1990.

[2] ISO/IEC JTC1/SC21/WG7 N838: Basic Reference Model of Open Distributed Processing-Part 1: Overview and Guide to Use. July, 1993.

[3] ISO/IEC CD 10746-2.3: Basic Reference Model of Open Distributed Processing-Part 2: Descriptive Model. July, 1993.

[4] ISO/IEC CD 10746-3.2: Basic Reference Model of Open Distributed Processing-Part 3: Prescriptive Model. July, 1993.

[5] ISO/IEC JTC1/SC21/WG7 N839: Basic Reference Model of Open Distributed Processing-Part 4: Architectural Semantics. July, 1993.

[6] Reference Model of Open Distributed Processing: A Tutorial. K. Raymond. CRC for Distributed Systems Technology, CITR, University of Queensland. Proceedings of 2nd ICODP, Berlin, 1993.

[7] OSF DCE Application Environment Specification/Distributed Computing RPC Volume. ISBN 0-13043688-7.

[8] X/Open Directory Service API Specification. X.400 API Association, X/Open Company Limited, 1990.

[9] ODP-Trader. M. Bearman. CRC for Distributed Systems Technology. Proceedings of 2nd International Conference on Open Distributed Processing, Berlin, 1993.

[10] Is DCE a Support Model for ODP? A. Beitz, P. King, K. Raymond. CRC for Distributed Systems Technology. Proceedings of 2nd International Conference on Open Distributed Processing, Berlin, 1993.

[11] Experiences with Building a Multimedia Mail System Prototype on the OSF DCE Platform. Information Architecture Group, Hewlett-Packard Document NSA-92-012. June, 1992.

[12] The DCE Experience. Information Architecture Group, Hewlett-Packard Document NSA-92-014. July, 1992.

[13] A Performance Study of the DCE 1.0.1 Cell Directory Service and Implications for Application and Tool Programmers, J. Martinka, et al. Lecture Notes in Computer Science 731, Springer-Verlag, 1993

[14] OODCE: A C++ Framework for the OSF Distributed Computing Environment. J. Dilley, HewlettPackard. To be published at Usenix 1995.

[15] A Distributed Object-Oriented Platform Based on DCE and C++. P. Bosco, G. Martini, C. Moiso, CSELT. Proceedings of 2nd International Conference on Open Distributed Processing, Berlin, 1993.

[16] Universal Networked Objects. J. Nichol, D. Curtis, D. Vines, N. Holt, O. Hurley, G. Lewis. OMG Document 94-9-32; Object Management Group, Inc. September, 1994. 